\title{
Comparison of Exercise and Physical activity routine and Health Status among Apparently Healthy Nigerian Adults Before and During COVID-19 Lockdown
}

Patrick Ayi Ewah ( $D$ payiewah8@yahoo.com )

University of Calabar https://orcid.org/0000-0002-6981-1288

Adetoyeje Y Oyeyemi

University of Maiduguri

Adewale L Oyeyemi

University of Maiduguri

Saturday N Oghumu

University of Calabar Teaching Hospital

\section{Peter Agba Awhen}

University of Calabar

Mary Ogaga

University of Calabar

Lucy Inyang Edet

University of Calabar

\section{Research Article}

Keywords: Exercise participation, Physical Activity, Perceived health, Covid-19 Pandemic, Lockdown

Posted Date: September 10th, 2021

DOI: https://doi.org/10.21203/rs.3.rs-873341/v1

License: @ (i) This work is licensed under a Creative Commons Attribution 4.0 International License. Read Full License 


\section{Abstract}

Background: COVID-19 is a pandemic that ravaged the world in manners that were never seen in the recent past and one of the measures to stem the tide-off this ravaging pandemic is a stay-at-home order referred to as lockdown. This study compares the physical activity status and perceived health of Nigerians before and during the lockdown.

\section{Results}

Social media platform users $(n=205)$ were surveyed using a two-part questionnaire. The $1^{\text {st }}$ part elicited the socio-demographic characteristics of the subject. In the $2^{\text {nd }}$ part, information about their exercise and physical activity, general health, and economic palliatives as offered by the government, non-governmental organizations, and philanthropists were elicited.

The frequency of exercise was significantly more $(\mathrm{p}<0.05)$ during the lockdown than before the lockdown. The duration and intensity of the exercise per week were comparable. There was also a negative relationship between the Body Mass Index, frequency, and duration of exercise before and during the lockdown. The subjects also perceived their health as worse during (3.70 \pm 1.05$)$ the lockdown than before $(3.95 \pm 0.97)$ lockdown $(Z=-3.69, p=0.00)$.

Conclusion: Overall, for these cohorts of social media platform users, lockdown did not adversely affect their exercise routine. It is recommended that there should be specific admonition on exercise as an important Instrumental Activity of Daily Living (IADL). Therefore, while this pandemic lockdown lasts and beyond, the safety measures to follow while partaking in this IADL, should be included in the public health recommendation.

\section{Background}

On the 28th of February, 2020, Nigeria announced the first confirmed case of COVID-19, the novel SARS-Cov-2 coronavirus ${ }^{1}$, and became the first West African country with a confirmed case of the novel COVID-19 ${ }^{2}$. Soon after the World Health Organization (WHO) declared that the novel coronavirus outbreak is a public health emergency of international concern ${ }^{3}$ the daily estimates were expected to rise 4,5 . Following the outbreak of the corona Virus pandemic, governments of different countries began to take swift, and protective measures to contain the spread, ${ }^{6}$ and in Nigeria, the National Center for Disease Control led the initiatives to manage the pandemic through measures including isolation, quarantine, testing, and treatment, and monitoring, and surveillance ${ }^{7}$.

In Nigeria, the administrative, and commercial capital cities of Lagos, and Abuja, and the state of Ogun in southwest Nigeria were placed on an initial two weeks lockdown. Subsequently, the details of COVID-19 measures were publicized, and the lockdown became extended to other cities, and towns in the states. The lockdown measures included, but are not exclusive to travel warnings, and bans, and flight cancellations, stay-at-home orders, closing down of both public, and private schools, closing down of houses of worships, and suggestions that workers on non-essential duties should work from home. Other details such as social distancing, frequent washing of hands or the use of alcohol-based sanitizers, wearing of face mask, avoidance of touching the eyes, nose, and mouth, were announced.

Health authorities advise people to stay at home although an average working Nigerian leaves home early in the morning daily in the week, and most of them walk for long or short distances, to work or to the farm, or to take transport to work. It is a general belief that staying at home can promote sitting or lying down for an extended period watching television, excessive use of mobile phones with possible deleterious effect on the health of such individuals, as a possible increase in sitting time and sedentariness, may worsen the health condition of those with a chronic condition, and may reduce the quality of life of even a healthy Nigerian adult 6,8 even when the lockdown is relaxed.

Regular participation in physical activity, and exercise (walking, jogging, cycling, and dancing) has a lot of health benefits ${ }^{9}$. Active living prevents diseases such as hypertension, arteriosclerosis, and diabetes ${ }^{10,11}$. It may also have other potential positive effects such as improved pulmonary function, and cardiovascular, and aerobic fitness, stronger immunity, enhanced muscle strength, higher bone density, stress reduction, and better emotional stability ${ }^{9}$. It is recommended that adults should undertake at least 30 minutes of moderate physical activity every day or at least 15 minutes of vigorous physical activity every day for at least 5 days a week ${ }^{12}$. 
Recent reports suggest that there is increasing awareness of the health-enhancing benefits of physical activity, and exercise among Nigerians. Anecdotal reports show that in many Nigerian cities in the morning, it is common to observe some young adults engaging in walking or jogging. It is the general belief that weekend mornings are especially the most common time people engage in exercise. Lack of exercise and hypo-activity can exacerbate any preexisting mental health condition such as anxiety, depression, and may lead to substance abuse, domestic violence, and sexual violations, and crimes ${ }^{13-14}$. Other than individual initiatives, and expert advice to media audiences, no advice or admonition on physical activity behavior during the pandemic, and especially during lockdown was issued by the National Center for Disease Control or any government agency in Nigeria.

Lockdown is a life-saving, and survival measure that is a necessity but it is a general belief that it impacts nations' economies negatively. The measure that restricts citizens to their homes also brings untold hardship to the general population. Many Nigerians were not able to earn any income, and palliatives are hardly enough succor where such is offered. Worldwide there is some consensus on the economic consequences of any lockdown. However, the health consequences of lockdown are not given much attention or are outrightly ignored. Lockdown restricts movement, and invariably also restricts the outdoor exercise, and activities in public parks, and fitness gyms. However, how the restrictions of movement during the lockdown due to COVID-19 affect Nigerian people is unclear, as there is hardly any empirical data on this especially in Nigeria.

The social media platform is today's forum of interaction by people of similar interests or dispositions. Social media platform users constitute a sizeable population of youths, and adults who are often highly educated and are homogenous groups constituting a significant sub-population of any nation in the contemporary world ${ }^{15}$. Through this platform, information is disseminated speedily among group members. Albeit inappropriately, this platform has become the means through which even official workplace communication is disseminated to employees, and health information including exercise, and physical activity instruction, and advice are shared through support groups via the social media platform ${ }^{16-17}$.

A physically active lifestyle is habituated and requires self-discipline to keep, and adherence to a physically active lifestyle is presumed to be low across the population especially in urban areas worldwide. Information on how the lockdown impacts the physical activity status of the populace is important to assess the possible overall effect of the lockdown on any population. How the lockdown measures in Nigeria impact the physical activity pattern or behavior of Nigerians is unclear. The objective of this study was therefore to compare the pattern, and level of physical activity behavior of Nigerians before, and during the period of lockdown. It was hypothesized that; there will be no significant difference between the frequency of exercise before, and during COVID-19 Lockdown, there will be no significant difference between the duration of exercise before, and during COVID-19 Lockdown, and there will be no significant relationship between the health status of the subject before, and during COVID-19 Lockdown.

\section{Methods}

\subsection{Subjects}

The subjects for this study were 18-60 years old. The recruited subjects, were Nigerians, can read, and write in English, and frequently make use of any of the social media (Facebook, WhatsApp, Messenger, or Link-in). The subjects were also residents in the country as of when the data was collected. Nigerians who are currently sick or under any form of treatment or medications for an ailment or who were hospitalized or on lockdown outside the country were excluded from participation.

\subsection{Instrumentation}

The main instrument for this study is a researcher-developed Physical Activity Survey on COVID-19 Lockdown. The first part of the 16-item survey sought sociodemographic information including age, occupation, and the current state of residence, self-reported height, and weight. In the second part, subjects were asked about their participation in physical activity, and exercise, and the type, duration, and frequency of exercise, and were also asked to rate themselves on their health status before, and during the COVID-19 lockdown. They were also asked about their level of satisfaction, and happiness or, unhappiness during the lockdown. Finally, they were asked whether they received any palliative measures from any government, non-government organization, or any individual philanthropist.

The instrument was reviewed by a university lecturer with not less than 10 years of experience in teaching, and research in physiotherapy, and community practice that includes instrument design, and adaptation. Five other professionals in the discipline of

Page 3/11 
physiotherapy with not less than 10 years of practice, and teaching experience attested to the face validity of the instrument. The reliability of the instrument was examined by checking internal consistency using the Cronbach alpha coefficient. Test-retest reliability and correlation analysis were also conducted for examining the reliability of the instrument. The internal consistency reflects the homogeneity of the questionnaire, with a Cronbach a of 0.60 . The instrument was tested for reliability by having 16 adults in the age range of $18-56$ complete the questionnaire using Spearman correlation ${ }^{18}$. The questionnaire was completed twice with a duration of one week between the first and second administration. There was a high degree of correlation between first, and second administration $(r=0.89, p<0.05)$. The questionnaire was tested using reliability analysis, and the spearman test at $a=$ 0.05 .

\subsection{Procedure}

Ethical approval was sought from the Research, and Ethical Committee of the University of Maiduguri, College of Medical Sciences, before the commencement of the study. In this online survey, the Physical Activity Survey on COVID-19 questionnaire was shared through social media platforms including Facebook, WhatsApp, Messenger, and Link-in platforms. Subjects who consent to participate were advised to click on a link that was sent and were advised to read the preliminary instructions before deciding to participate in the survey.

In the preliminary instruction, subjects were invited to participate. Invited subjects were informed that participation is voluntary and that no name was required on the survey. In addition to assuring them of anonymity, they were also informed that any data obtained will be pooled together with those of others, and will be treated in aggregates only and that no harm will be sustained during participation.

In addition to previous information, prospective subjects were informed that if they consent to participate, the survey should be completed and that they can decline participation by not completing the survey without any punishment or penalty. No gifts or reward was pledged for the subjects, but the subjects were informed that the results of the study will be shared with anyone who indicated interest, when appropriate, and only upon request. Upon return of the survey questionnaire, all completed, and usable responses were entered into a computer for statistical analysis. Parametric and nonparametric data were treated as appropriate for descriptive, and inferential analysis of the data.

\section{Data Analysis}

Data obtained were entered into a computer and were analyzed using the Statistical Package for Social Scientists (SPSS, version 20). Descriptive statistics of mean, and standard deviation, and frequencies were used to summarize the sociodemographic, and physical characteristics of the data. The data were subjected to a test for normality using Kolmogorov-Smirnov test, and normally distributed variables were analyzed with paired t-test (for differences), and Person's correlation (relationship test), and non-normal data were analyzed using spearman's correlation, Wilcoxon signed-rank, and Chi-square (test for a relationship, difference, and association). These analyses were used to explore trends of relationship, associations, and the difference between the frequency, intensity, and time duration of exercise. As well as other applicable data on participation in physical activity, and the perceived health status of subjects before, and during COVID-19 lockdown. The level of significance was set at $p<0.05$.

\section{Results}

\subsection{Physical Characteristics of the subjects}

A total of 205 subjects who were physiotherapists, medical laboratory scientists, Civil servants, students, housewives, and businessmen participated in the study. A simple majority of the subjects were male $(53.3 \%, n=110)$, and $45.9 \%(n=95)$ were female. The mean age, weight, and height of the subjects were $31.79 \pm 8.38$ years, $70.52 \pm 15.33 \mathrm{~kg}$, and $1.68 \pm 0.12 \mathrm{~m}$ respectively. A simple majority $(49.3 \%)$ of the subjects were civil servants $(n=101)$. The mean duration, the subjects used to complete the electronic survey was $12.18 \pm 18.66$ minutes Table 1 . Seventy-one (34.6\%) of the subject engaged in walking exercise, and $30.2 \%$ engaged in jogging $(n=62)$. Some subjects about $22.9 \%(n=47)$ engaged in a combination of aerobic, and weight lifting, abdominal exercises, or play sports like badminton. Only a few of the subject engaged in dancing $(n=16)$, skipping $(n=5)$, cycling $(n=3)$, and swimming $(n=1)$. 
A majority of the subject either strongly disagree $(n=83)$ or disagree $(n=70)$ that they are happy that they were in a lockdown, and $3.9 \%(n=8)$ either strongly agree or agree $(21.5 \% ; n=44)$. A simple majority of the subject also strongly disagree $(n=102)$ or disagree $(n=62)$ that they are happy with the palliative measures provided by the government while others agree $(n=35)$ or strongly agree $(n=6)$ that they are happy. Also, a majority of the subject strongly disagree or disagreed $(n=183)$ that they had received any of these palliative measures provided during the lockdown while only a few either agreed or strongly agreed $(n=22)$. Majority of the subjects were of ages $18-30(n=97)$, followed by $31-40(n=80), 41-50(n=24)$ and $51-60(n=4)$.

\subsection{Comparison, relationship, and association before, and during COVID- 19 Lockdown}

The number of days during which the subjects exercise or engage in physical activity was significantly higher $(p=0.03)$ during the lockdown than before the lockdown ( $3.32 \pm 2.01$ vs. $3.04 \pm 1.78$ days), but there was no difference in duration of exercise $(p=0.10)$ before $(37.27 \pm 33.89)$, and during $(33.59 \pm 29.20)$ the COVID19 lockdown. There was however significant strong, positive relationship between the duration of exercise before, and during $(r=0.50 ; p=0.00)$. A significant strong relationship was also found between the frequency of exercise before and during the lockdown $(r=0.53 ; p=0.00)$. On the contrary, a significant medium positive relationship was found between the intensity of exercise before, and during the lockdown $(r=0.39 ; 0.00)$. There was also a negative relationship (not significant) between the BMI and duration of exercise before and during the lockdown (Table 2). Hence, an increase in BMI results in a decrease in the duration, frequency, and intensity of exercise albeit not to a significant level. The perceive health of subjects was significantly better $(Z=-3.69, p=0.00)$ before $(H B L D=3.95 \pm 0.97)$ the lockdown than during $(H D L D=3.70 \pm 1.05)$ the lockdown. There was also a small negative (not significant) relationship between being Happy with Lockdown - health Before Lockdown, Happy with Palliative Measure - Health During Lockdown, and Receive Palliative Measure/health before, and during the lockdown, however, there was a small negative but significant relationship $\left(r=-0.15 ; p=0.03^{*}\right)$ between being Happy with Palliative Measure -Health Before Lockdown, relationships between other variables can be seen in Table 3. There was also a highly significant association between HappLD - HappPM $(p=0.00)$, HappLD - RecPM $(p=0.00)$, HappPM - RecPM $(p=0.00)$, and HBLD - HDLD ( $p=$ $0.00)$ (Table 4). However, there was no significant association ( $p>0.05)$ between HappLD - HBLD; HappLD - HDLD; HappPM HBLD/HDLD (Table 4).

\section{Discussion}

Lockdown is a potent measure of saving a life that was employed throughout the world, and many countries (China, Korea, Italy, US) in the forefront of the pandemic adopt this measure, one way or the other either wholly or partially ${ }^{19,14}$. Lockdown, although a lifesaving measure (with success in containing COVID-19). The measure impacts negatively on the nation's economy, and in turn the individuals, as it creates untold hardship, and dwindles the ability to earn income ${ }^{20}$ which may be implicated for the poor overall health as reported in the present study. This study set out a tone on how a stay-at-home order by the government impacts the lifestyle of its citizenry.

A Majority of the subjects in the present study were of the young adult population between 18-30 years is consistent with the findings of Ngnoso ${ }^{15}$, which attest to the fact that social media platform users constitute a sizeable population of the young adult. The finding in the present study is also consistent with that of Jan Hruska, and Maresova ${ }^{21}$ who found out that the above age group made use of social media more frequently, and that as age increases the number of users of social media decreases.

The subjects in this study performed exercise more frequently during the lockdown than before the lockdown but there was no difference in the duration, and intensity of the exercise. On average lockdown duration only modestly but insignificantly exceeded the recommendation on physical activity ${ }^{12}$. Increased frequency of exercise during as against before the COVID-19 Lockdown as seen in the present study may be attributed to the fact that subjects were using coping strategies to avoid the deleterious effect of lockdown. A strong positive relationship between duration, and frequency of exercise before, and during the COVID-19 lockdown, signifies that an increase in the frequency of exercise before COVID-19 lockdown also increases duration and frequency of exercise during the lockdown. A negative relationship (non-significant) between BMI, duration, frequency, and intensity of exercise before, and during COVID-19 lockdown as reported in the present study, signifies that as BMI increases the duration, frequency, and intensity of exercise also reduces concurrently albeit not to a significant level. It has been shown that an increase in the frequency and duration of physical activity resulted in greater weight loss, fat loss, and reductions in measures of central obesity 22 . 
Hence we inferred that subject with higher BMI tends to spend less duration, intensity, and frequency during exercise before, and during the lockdown.

Lockdown especially during a global pandemic like COVID-19 turns a once energetic individual into becoming hypoactive and reduced the quality of life of even the healthiest individual hence worsening chronic condition, ${ }^{9,7}$ such individuals may be left with no option than increasing the number of days in a week they engage in exercise. Our hypothesis that there will be no difference in the frequency of exercise before, and during the pandemic lockdown is therefore rejected.

Increasing the frequency of exercise during the lockdown may be regarded as a coping mechanism. However, unfortunately, this coping mechanism did not prevent the feeling of poor health during the lockdown perhaps due to the modesty of the improvement in the frequency which is not enough to meet the health-enhancing exercise, and physical activity recommendation of at least 5 days a week, and 30 minutes per session ${ }^{12}$. It could also be that the magnitude of the impact of the lockdown on health is too much more than a modest improvement in exercise participation could counteract. The finding in the present study is consistent with a previous study by Grasdalsmoen et.a ${ }^{23}$ which concluded that a large majority of young adults fail to meet international recommendations on exercise.

The modest but insignificant improvement in the duration of participation in physical activity is good but it still could not have resulted in improved well-being or health by the subjects. This finding is therefore not in accord with the consensus that increased participation in physical activity should lead to an improvement in perceived well-being, and overall health status (psychological well-being) of subjects ${ }^{24}$. Our hypothesis that there would be no significant difference in duration of exercise before, and during the lockdown is therefore not rejected.

It is also notable that the overall health perception is worse during the pandemic lockdown than it was before the lockdown. On a good note, however, this study suggests that there may not be any recidivism in the exercise habit cultivated before the lockdown, and during the pandemic lockdown among this cohort of social media platforms users. A significantly poorer health status during the lockdown than before the lockdown as observed in the present study is consistent with finding by Lima, et al. ${ }^{20}$, who reported that measure which disrupts people's jobs, and lives could take a heavy toll on people's health, and well-being. Also, our hypothesis that there will be no significant difference in the health status before, and during the lockdown is therefore rejected.

Exercise and health-enhancing physical activity are important IADL that is recommended for all adults to stem the tide, off chronic lifestyle diseases such as; diabetes, obesity, and heart diseases, which is now regarded as a pandemic even worldwide. It is unknown whether the subjects in this study exercise in groups, alone, indoor in the gym or they exercise outdoor. Nonetheless, it is important to include social distancing, and other measures such as enforced compliance with gym occupancy rate when exercising indoor, avoiding overcrowding indoor, and outdoor as well as observe other measures to prevent the spread of any COVID-19, and infectious diseases in the context of the current pandemic.

\subsection{Study Limitations}

The result of this study should be interpreted in place of some limitations. First, the use of cross-sectional data, which precludes establishing a temporal, and causal comparison between physical activity, and the rating of perceived health among this cohort of social media users. Second, the self-reported value on height, weight, and health may not reflect true measure as subjects may not know their actual values on the physical characteristics. Lastly, exercise frequency and durations are estimates and may be more or less than the values reported. Never the less this study provide some insights into the experience of a cohort of social media platform users that may be fairly generalizable to a not insignificant subpopulation of Nigerians, during the COVID - 19 pandemic.

\subsection{Conclusions}

The present study showed that the number of days in which subjects engage in physical activity was significantly higher during the COVID-19 lockdown than before the lockdown, but no difference was observed between the duration of exercise before and during the lockdown. The subject in the present study also reports significantly poorer health during the COVID-19 lockdown than before the lockdown, and there is a highly significant association between I have received palliative during the lockdown and being happy during the lockdown. 


\section{Abbreviations}

COVID = Corona Virus Disease BMI= Body Mass Index, IADL= Instrumental Activity of Daily Living WHO= World Health Organization, DEXB/DLD = Duration of Exercise Before and During Lockdown; FEXB/DLD= Frequency of Exercise Before and During Lockdown; INTEXBLD = Intensity of Exercise Before and During Lockdown, HappLD= Happy with lock down; HappPM=Happy Palliative Measure; RecPM= Receive Palliative Measure; HBLD/HDLD= Health Before and During Lockdown

\section{Declarations}

\section{Ethics approval and consent to participate}

Ethical approval was sought from the Research, and Ethical Committee of the University of Maiduguri, College of Medical Sciences, before the commencement of the study and informed consent was sought from individual subjects.

\section{Consent for publication}

Not applicable

\section{Availability of data and material}

Data and material are available upon request.

\section{Competing interests}

Authors declare no competing interest.

\section{Funding}

No funding for these study.

\section{Authors' contributions}

Patrick PA conceived the idea \& is the principal investigator. Adetoyeje Y O, and Adewale LO, supervised the work and contributed in questionnaire design. Oguhumu NS, Mary O, Lucy IE, and Awhen PA edited the manuscript. All authors contributed in preparing and writing the manuscript.

\section{Acknowledgements}

None.

\section{Conflict of interest}

The authors declare has no conflict of interest associated with this paper.

\section{References}

1. Lai CC, Shih TP, Ko WC, et al. Severe acute respiratory syndrome coronavirus 2 (SARS-CoV-2) and coronavirus disease- 2019 (COVID-19): The epidemic and the challenges. Int J Antimicrob Agents 2020;55:105924. DOI: 10.1016/j.ijantimicag. 2020.105924.

2. Ruth Olurounbi and Elisha Bala-Gbogbo, 2020. Africa's Biggest City on Lockdown to Curb Virus in Nigeria https://www.bloomberg.com/news/articles/2020-03-29/nigeria-imposes-lockdown-on-its-two-main-cities-ogun-state March 29, 2020, 7:35 PM GMT+1 Updated on March 29, 2020, 8:01 PM GMT+1

3. World Health Organization. Statement on the second meeting of the International Health Regulations (2005) Emergency Committee regarding the outbreak of novel coronavirus (2019-nCoV). Available at: https://www.who. int/news-room/detail/30-012020-statement-on-the-second-meeting-of-theinternational- health-regulations-(2005)-emergency-committee-regarding-theoutbreakof-novel-coronavirus-(2019-ncov). [accessed 30.01.2020]. 
4. Health Emergency Office, National Health Commission of the People's Republic of China. Outbreak notification: update on pneumonia of new coronavirus infection as of 24:00 on January 30. Available

at:http://www.nhc.gov.cn/xcs/yqtb/202001/a53e6df293cc4ff0b5a16ddf7b6b2b31.shtml. [accessed 31.01.2020]. [in Chinese].

5. Johns Hopkins University. Coronavirus 2019-nCoV Global Cases by Johns Hopkins CSSE. Available at: https://gisanddata.maps.arcgis.com/apps/opsdashboard/index.html\#/bda7594740fd40299423467b48e9ecf6. [accessed 31.01.2020].

6. Chen P, Mao L, Nassis GP, Harmer P, Ainsworth BE, Li F. Coronavirus disease (COVID-19): The need to maintain regular physical activity while taking precautions. J Sport Health Sci 2020;9:103_4.(http://creativecommons.org/licenses/by-nc-nd/4.0/). https://doi.org/10.1016/j.jshs.2020.02.001

7. Nigerian Centre for Disease Control, 2020. 29th February 2020| PUBLIC HEALTH ADVISORY TO NIGERIANS ON NOVEL CORONAVIRUS (\#4)https://ncdc.gov.ng/news/234/29th-february-2020\%7C-public-health-advisory-to-nigerians-on-novel-coronavirus$\% 28 \% 234 \% 29$

8. Owen N, Sparling PB, Healy GN, Dunstan DW, Matthews CE. Sedentary behavior: emerging evidence for a new health risk. Mayo Clin Proc 2010;85:1138-41.

9. Hyun-Gyu Cha, Tae-Hoon K., \& Myoung-Kwon K. Therapeutic efficacy of walking backward and forward on a slope in normal adults. J. Phys. Ther. Sci. 28: 1901-1903, 2016

10. Fletcher GF, Balady G, Blair SN, et al. Statement on exercise: benefits and recommendations for physical activity programs for all Americans. Circulation. 1996;94:857-862. [PubMed] [Google Scholar]

11. National Institutes of Health. Clinical guidelines on the identification, evaluation, and treatment of overweight and obesity in adults: the evidence report. In: Panel E, ed.

NHLBI Obesity Education Initiative Expert Panel on the Identification, Evaluation, and Treatment of Overweight and Obesity in Adults. Bethesda, Md: National Institutes of Health; 1998.

12. U.S. Department of Health and Human Services. Physical activity guidelines for Americans. 2nd ed. Washington, DC: U.S. Department of Health and Human Services; 2018.

13. Cao W., Fang, G. Hou, et al., (2020): The psychological impact of the COVID-19 epidemic on college students in China. Psychiat.Res.(2020), 10.1016/j.psychres.2020.112934

14. Zhang S X, Yifei Wang, Andreas R, Feng Wei, et al., (2020): Unprecedented disruption of lives: health, distress and life satisfaction of working adults in China one month into the COVID-19 outbreak: Psychiatry Research vol.288,112958; https;//doi.org/10.1016/j.psychrea.2020.112958.

15. Ngonso BF. Effect of Social Media on Teenagers and Youths: A Study of Rural Nigerian Teenagers and Youths in Secondary Schools. Global Media Journal 2019, 17:32.

16. Leonardi, Paul M., Marleen Huysman, Charles Steinfield, Enterprise Social Media: Definition, History, and Prospects for the Study of Social Technologies in Organizations, Journal of Computer-Mediated Communication, Volume 19, Issue 1, 1 October 2013, Pages 1-19, https://doi.org/10.1111/jcc4.12029

17. Baruah, Trisha Dowerah: Effectiveness of Social Media as a tool of communication and its potential for technology-enabled connections: A micro-level study. International Journal of Scientific and Research Publications, Volume 2, Issue 5, May 20121 ISSN 2250-3153 www.ijsrp.org.

18. Twee CB, Bot SDM, Boer, MR et al., (2007): Quality criteria were proposed for measurement properties of health status questionnaire. J Clin Epidemiol 60(1):34-42.d0i:10.1016/j.jclinepi.2006.30.012 
19. Moodie Davitt Report, 2020. COVID-19 Update: Encouraging numbers from Korea; Italian lockdown; Japan suspends visas for Chinese and Korean visitors. https://www.moodiedavittreport.com/covid-19-update-encouraging-numbers-from-koreaitalianlockdown-japan-suspends-visas-for-chinese-and-korean-visitors/ (accessed 8 March 2020).

20. Lima, C.K.T, de Medeiros Carvalho, P.M., Lima, et al., (2020): the emotional impact of coronavirus 2019-Nov (new coronavirus Disease). Psychiat.Res. (20200, 10.1016/j.psychres.2020.112915.

21. Jan Hruska and Petra Maresova, (2020): Use of Social Media Platforms among Adults in the United States-Behavior on Social Media; Societies 2020, 10, 27; doi:10.3390/soc10010027 www.mdpi.com/journal/societies

22. Slentz CA, Duscha BD, Johnson JL, Ketchum K, Aiken LB, Samsa GP, Houmard JA, Bales CW, Kraus WE. Effects of the amount of exercise on body weight, body composition, and measures of central obesity: STRRIDE--a randomized controlled study. Arch Intern Med. 2004 Jan 12;164(1):31-9. doi: 10.1001/archinte.164.1.31. PMID: 14718319.

23. Grasdalsmoen M, Eriksen HR, Lønning KJ, Sivertsen B. Physical exercise and body-mass index in young adults: a national survey of Norwegian university students. BMC Public Health. 2019;19(1):1354. Published 2019 Oct 23. doi:10.1186/s12889-0197650-z

24. Butt, Z. I, Rashid, K., Mahanoor, Akhtar T, Saeed N.1, Muhammad Abdul Jabar Adnan M.A. Shamsa Hashmi S. (2016): RELATIONSHIP BETWEEN PSYCHOLOGICAL WELL BEING AND EXERCISE; Sci.Int.(Lahore),28(2),1569-1574,2016 ISSN 1013-5316; CODEN: SINTE 8 1569. Available from: https://www.researchgate.net/publication/301768840_RELATIONSHIP_BETWEEN_PSYCHOLOGICAL_WELL_BEING_AND_EXERCISE [accessed Nov 28 2020].

\section{Tables}

Table 1: Physical Characteristics of the Subjects (values are in mean and SD)

\begin{tabular}{|c|c|c|}
\hline Variables & Mean/SD & CV \\
\hline Age (years) & $31.79 \pm 8.38$ & 0.26 \\
\hline Weight in kilogram (kg) & $70.52 \pm 15.33$ & 0.22 \\
\hline Height meter (m) & $1.68 \pm 0.12$ & 0.07 \\
\hline Body Mass Index $\left(\mathrm{kg} / \mathrm{m}^{2}\right)$ & $25.18 \pm 5.76$ & 0.23 \\
\hline Frequency of exercise before lockdown (days/week) & $3.04 \pm 1.78$ & 0.59 \\
\hline Freq. Exercise during the lockdown (days/week) & $3.32 \pm 2.01$ & 0.61 \\
\hline Duration Exercise before the lockdown (minutes/day) & $37.27 \pm 33.89$ & 0.91 \\
\hline Duration Exercise during the lockdown (minutes/day) & $33.59 \pm 29.20$ & 0.87 \\
\hline Intensity of exercise before the lockdown (minutes/week) & $135.52 \pm 183.63$ & 1.36 \\
\hline Intensity of exercise during the lockdown (minutes/week) & $128.55 \pm 137.35$ & 1.07 \\
\hline Completion time (minutes) & $12.18 \pm 18.66$ & 1.53 \\
\hline
\end{tabular}

$\mathrm{CV}=$ Coefficient of Variation (Standard Deviation divided by mean) 
Table 2: Relationship between Body Mass Index, Duration of exercise before and during Lockdown, frequency of exercise before and during, and Intensity of exercise before and during COVID-19 Lockdown

\begin{tabular}{lcl} 
Variables & r-values & p-values \\
\hline BMI $\left(\mathrm{kg} / \mathrm{m}^{2}\right)$-DEXBLD & -0.01 & $0.86^{\mathrm{NS}}$ \\
BMI $\left(\mathrm{kg} / \mathrm{m}^{2}\right)$-DEXDLD & -0.09 & $0.21^{\mathrm{NS}}$ \\
BMI $\left(\mathrm{kg} / \mathrm{m}^{2}\right)$-FEXBLD & -0.04 & $0.60^{\mathrm{NS}}$ \\
BMI $\left(\mathrm{kg} / \mathrm{m}^{2}\right)$-FEXDLD & -0.10 & $0.14^{\mathrm{NS}}$ \\
BMI $\left(\mathrm{kg} / \mathrm{m}^{2}\right)$-INTEXBLD & -0.02 & $0.74^{\mathrm{NS}}$ \\
BMI $\left(\mathrm{kg} / \mathrm{m}^{2}\right)$-INTEXDLD & -0.07 & $0.35^{\mathrm{NS}}$ \\
\end{tabular}

DEXB/DLD = Duration of Exercise Before and During Lockdown; FEXB/DLD= Frequency of Exercise Before and During Lockdown; INTEXBLD = Intensity of Exercise Before and During LockDown; BMI $\left(\mathbf{k g} / \mathbf{m}^{2}\right)=$ Body Mass Index in Kilogram; NS= not significant

Table 3: Relationship between happy with Lockdown, happy with Palliative Measure, Receive Palliative Measure, Health Before and During COVID-19 Lockdown

\begin{tabular}{lcc} 
Variables & r-values & p-values \\
\hline Happy with Lockdown-Happiness Palliative measure & 0.32 & $0.00^{* *}$ \\
Happy with Lockdown-Receive Palliative measure & 0.22 & $0.00^{* *}$ \\
Happy with Lockdown-health Before Lockdown & -0.09 & $0.20^{\mathrm{NS}}$ \\
Happy with Lockdown- Health During Lockdown & 0.18 & $0.01^{*}$ \\
Happy with Palliative Measure-Receive Palliative measure & 0.54 & $0.00^{* *}$ \\
Happy with Palliative Measure- Health before Lockdown & -0.15 & $0.03^{*}$ \\
Happy with Palliative Measure- Health during Lockdown & -0.04 & $0.62^{\mathrm{NS}}$ \\
Receive Palliative Measure- Health before Lockdown & -0.14 & $0.05^{\mathrm{NS}}$ \\
Receive Palliative Measure- Health during Lockdown & -0.05 & $0.51^{\mathrm{NS}}$ \\
Health before Lockdown- Health during Lockdown & 0.51 & $0.00^{* *}$ \\
\end{tabular}

**= highly significant $\mathrm{p}<0.01$ level (2-tailed); * = significant at $\mathrm{p}<0.05$ level ( 2 -tailed); NS= not significant $\mathrm{p}>0.05$ 
Table 4: Association between happy with Lockdown, happy with Palliative Measure, Receive Palliative Measure, Health Before and During COVID-19 Lockdown

\begin{tabular}{lccccc}
\hline Variables & values & df & Phi & Cramer's V & p-values \\
\hline HappLD - HappPM & 60.13 & 9 & 0.54 & 0.31 & $0.00^{*}$ \\
HappLD - RecPM & 24.10 & 9 & 0.34 & 0.20 & $0.00^{*}$ \\
HappLD-HBLD & 15.95 & 12 & 0.28 & 0.16 & $0.19^{\mathrm{NS}}$ \\
HappLD- HDLD & 14.74 & 12 & 0.27 & 0.16 & $0.26^{\mathrm{NS}}$ \\
HappPM-RecPM & 145.00 & 9 & 0.84 & 0.49 & $0.00^{*}$ \\
HappPM - HBLD & 16.91 & 12 & 0.29 & 0.17 & $0.15^{\mathrm{NS}}$ \\
HappPM- HDLD & 19.47 & 12 & 0.31 & 0.18 & $0.08^{\mathrm{NS}}$ \\
RecPM- HBLD & 16.41 & 12 & 0.29 & 0.16 & $0.17^{\mathrm{NS}}$ \\
RecPM-HDLD & 10.16 & 12 & 0.22 & 0.13 & $0.60^{\mathrm{NS}}$ \\
HBLD- HDLD & 170.00 & 16 & 0.91 & 0.46 & $0.00^{*}$ \\
& & & & & \\
\hline
\end{tabular}

HappLD = Happy with lock down; HappPM=Happy Palliative Measure; RecPM= Receive Palliative Measure; HBLD/HDLD $=$ Health Before and During Lockdown; ${ }^{*}=\mathrm{p}<0.05$; NS= $\mathrm{p}>0.05$. 